\title{
NAVEGAÇÂO DE ROBÔS MÓVEIS PARA MISSÕES DE BUSCA E VIGILÂNCIA BASEADA EM VOOS DE LÉVY
}

LEONARDO DE A. FREDERICO ${ }^{1}$, LUIZ DE SIQUEIRA MARTINS FILHO ${ }^{2}$.

${ }^{1,2}$ CECS/UFABC-Centro de Engenharia, Modelagem e Ciências Sociais Aplicadas - Universidade Federal do ABC. Av. dos Estados, 5001. Bairro Bangu. Santo André - SP - Brasil CEP 09210-580

$$
\text { leo_de_avellar@hotmail.com }{ }^{1} \text { luiz.martins@ufabc.edu.br }{ }^{2}
$$

\begin{abstract}
In this work, we study the problem of mobile robot navigation in terrain exploration missions for the purpose of surveillance or search. In these cases, the most desirable features for the trajectories are unpredictable of movement and fast full scan of the surveillance robot workspace. The proposed strategy can also be interesting for soil cleaning tasks, especially if the terrain map is not available. In this kind of tasks, the path planning for full scan can be difficult,and consequently an unplanned move can be an efficient solution. The proposed solution uses random walks that ensures the full terrain scan and at the same time, it not allows a prevision or anticipation of paths by a potential intruder. A particular type of random motion with characteristics that resemble the animals trajectories in search of food is called Lévy flight. This paper presents a pre-study of using Lévy flight on navigation control robot.
\end{abstract}

Keywords-Mobile robots, Trajectories planning, Lévy flights.

\begin{abstract}
Resumo-Nesse trabalho, estuda-se o problema de navegação de robôs móveis em missões de exploração de terrenos com o propósito de realizar busca ou vigilância. Nesses casos, as características mais desejáveis para as trajetórias são a imprevisibili dade de movimento e a rápida e completa varredura do espaço de trabalho do robô vigilante. A estratégia proposta também pode ser interessante para missões de limpeza do solo, especialmente se o mapa do terreno não está disponível. Para esse tipo de tarefa, o planejamento da trajetória para a varredura completa pode ser difícil, e consequentemente, um movimento não planejado pode ser uma solução eficiente para o problema. A solução proposta utiliza movimentos aleatórios, de modo a garantir a varredura completa do terreno e ao mesmo tempo não permitir uma previsão ou antecipação das trajetórias por parte de um eventual intruso. Um tipo particular de movimento aleatório com características que se assemelham às trajetórias de animais na busca por alimentos é o chamado voo de Lévy. Esse artigo apresenta um pré-estudo para a utilização do voo de Lévy como controle de navegação de um robô vigilante.
\end{abstract}

Palavras-chave— Robôs móveis, Planejamento de trajetórias, Voo de Lévy.

\section{Introdução}

O vôo de Lévy é um movimento aleatório (random walk) baseado na função densidade de probabilidade de Lévy (proposta pelo matemático francês Paul Pierre Lévy), cujo padrão de trajetórias é caracterizado por seguidos passos (descolamentos retílineos) de comprimentos curtos intercalados por um passo de comprimento longo. O vôo de Lévy tem sido mostrado como uma solução com características de otimização para o problema da busca por um determinado alvo (uma presa, uma fonte de alimento, um indivíduo, um hospedeiro) quando não é conhecida a posição do mesmo, conforme em Viswanathan (2008) e Nurzaman (2009). No vôo de Lévy,a cada passo do processo, uma trajetória é realizada, cujo valor de comprimento é escolhido a partir de uma função densidade de probabilidade $\mathrm{f}(\mathrm{x})$ que possui uma lei de potência de cauda longa (power-law long-tail) dada por:

$$
f(x)=\sqrt{\left(\frac{c}{(2 \pi)}\right)} *\left(\frac{\left(\left(\exp \left(\frac{(-c)}{(2(x-\mu))}\right)\right)\right)}{(x-\mu)^{(3 / 2)}}\right)
$$

onde c é o parâmetro de escala da função e $\mu$ é o parâmetro de localização. O primeiro indica o valor do decaimento da função com a aumento do comprimento, e o segundo indica o valor mínimo da variável independente (comprimento de trajetória).

A eficiência do vôo de Lévy e seu padrão que muito se assemelha ao observado no movimento de busca de certos animais, conforme em Edwards (2007), podem levar a considerá-lo como sendo uma solução ótima ou sub-ótima, podendo ser oriunda de interações complexas com o meio ambiente, Viswanathan (2008). Algumas soluções propostas para esse problema baseiam-se na aplicação de dinâmicas de sistemas caóticos para produzir as características desejadas para o movimento do robô vigilante, Martins-Filho (2004), Martins-Filho (2001), Nakamura (2001). 


\section{Planejamento das Trajetórias}

O objetivo deste trabalho é estudar o vôo de Lévy e sua implementação em um robô para missões de busca e vigilância. Foram realizadas simulações numéricas utilizarando a plataforma e a linguagem do software MATLAB ${ }^{\circledR}$. Com base na função de distribuição de Lévy, o algoritmo realiza um pseudo-sorteio para a escolha do comprimento da trajetória em um certo caminho do robô. O ângulo tomado para o caminho é dado segundo uma distribuição uniforme. Uma vez que o vigilante deve estar dentro de uma área delimitada (aqui considerada um quadrilátero de $500 \mathrm{~m}$ de lado), seu caminho em um dado instante deve sofrer certos desvios. A heurística adotada para esta restrição é a seguinte: quando o caminho do robô excede o limite da área de vigilância, este realiza o movimento até o ponto limite, sendo que o restante do comprimento não percorrido é percorrido voltando-se para dentro da área, e o ângulo para este movimento é oposto ao anterior. A fig. 1 exemplifica esse comportamento:

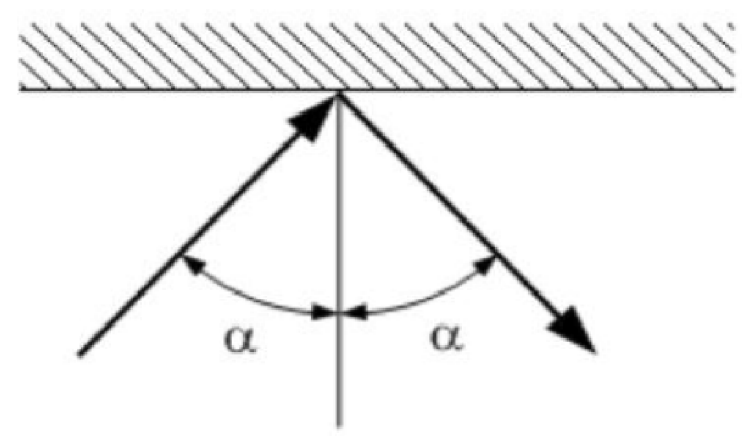

Figura 1: Mudança de direção do movimento quando o robô atinge o limite do espaço de trabalho.

O movimento do robô é realizado a partir do pseudo-sorteio da variável aleatória do comprimento, segundo a função densidade de probabilidade de Lévy, e do pseudo-sorteio do valor do ângulo da trajetória, segundo à função densidade de probabilidade uniforme (ângulo entre 0 e $2 \pi$ ). $O$ próximo ponto do robô é calculado por:

$$
\begin{aligned}
& X_{i+1}=X_{i}+f\left(x_{i}\right) \cos \left(\theta_{i}\right) \\
& Y_{i+1}=Y_{i}+f\left(x_{i}\right) \sin \left(\theta_{i}\right)
\end{aligned}
$$

onde $\left(\mathrm{X}_{\mathrm{i}}, \mathrm{Y}_{\mathrm{i}}\right)$ indica a posição atual e $\left(\mathrm{X}_{\mathrm{i}+1}, \mathrm{Y}_{\mathrm{i}+1}\right)$ indica o próximo ponto da posição robô .

A intenção de prover ao vigilante este comportamento é de não perder a proximidade com o comportamento de um movimento livre (em ambos os casos, o comprimento total percorrido é o mesmo). A partir disso, foi possível verificar as trajetória do vigilante, a partir de um ponto inicial, por todo o terreno. $\mathrm{O}$ terreno foi dividido em vinte e cinco sub-terrenos. A cada metro percorrido, a posição do vigilante no terreno é contada, de forma a indicar quantas vezes o robô passou por aquele sub-terreno. A partir disso, desejou-se relacionar a distribuição de cobertura aos parâmetros da função de Lévy e ao perfil de trajetórias exibidas pelo vigilante. Considerando o efeito de repetibilidade dos caminhos para a trajetória delimitada do vigilante, desejou-se também verificar a relação deste comportamento com os parâmetros da função de Lévy e a distribuição de caminhos no terreno.

\subsection{O Robô Móvel}

O robô móvel considerado nesse trabalho é um típico robô de movimento diferencial com 2 graus de liberdade, composto por 2 rodas ativas, paralelas e independentes, uma terceira roda passiva tem a função exclusivamente de equilíbrio do robô (um tipo de roda livre ou rodízio), e sensores de proximidade com capacidade de detecção de obstáculos. As rodas ativas são independentemente controladas na velocidade e no sentido de rotação. Os sensores proveem distâncias a obstáculos próximos, basicamente sensores de infravermelho (a fonte de luz está instalada no próprio sensor. Adicionalmente, consideramos que o robô está equipado com sensores específicos que permitem detectar e reconhecer os objetos procurados ou intrusos. Esse modelo de robô representa um compromisso interessante entre simplicidade no controle e graus de liberdade que permitem cumprir os requisitos de mobilidade. Além disso, ele é amplamente adotado em muitas pesquisas em robótica móvel. $\mathrm{O}$ corpo do robô é considerado como sendo rígido, e operando no plano horizontal. Seu movimento é obtido pelo comando das rodas ativas. O movimento resultante é descrito em termos da velocidade do centro de um ponto central do robô (e.g., o ponto médio do eixo entre as 2 rodas), $v(t)$, e a direção do robô, $\theta(t)$, ou movimento rotacional do robô em torno desse mesmo ponto médio, $\omega(t)$.

O controle de movimento do robô pode ser dado fornecendo as velocidades das 2 rodas, $\omega l(t)$ and $\omega r(t)$, ou, de maneira equivalente, a velocidade do ponto central e da velocidade angular do corpo, $v(t)$ and $\omega(t)$. Essas variáveis são chamadas de variáveis de controle. O modelo matemático desse problema de cinemática considera, além das variáveis de controle, outras 3 variáveis de estado: a posição e a orientação do robô $(x(t), y(t), \theta(t))$, Siegwart (2004).

$$
\left|\begin{array}{c}
\dot{x} \\
\dot{y} \\
\dot{\theta}
\end{array}\right|=\left|\begin{array}{cc}
\cos (\theta) & 0 \\
\operatorname{sen}(\theta) & 0 \\
0 & 1
\end{array}\right|\left|\begin{array}{c}
v \\
\omega
\end{array}\right|
$$

Essas equações constituem um sistema dinâmico não holonômico. O controle desse sistema tem sido intensivamente por diversos pesquisadores de e diversas soluções estão apresentadas na literatura. A estratégia 
de controle a ser adotada nesse projeto envolverá uma lei de controle baseada na realimentação de estado, que é adequada para obter uma trajetória desejada através de uma sequência de pontos $(x G, y G)$. Isso significa que uma tarefa de planejamento de trajetória deverá ser incorporada na solução, para fornecer essa sequência de pontos a ser seguida pelo robô, e que deverá respeitar as informações sobre obstáculos.

\section{Resultados e Discussões}

Para todas as simulações feitas, foi definido que o parâmetro de localização $\mu$ seria nulo. Isso teve não só o efeito de simplificar a função de Lévy, mas principalmente garantir a escolha de qualquer valor de trajetória para o vigilante numa certa iteração (já que o parâmetro de localização representa o valor mínimo de um deslocamento). Dessa forma, as análises das trajetórias do vigilante foram feitas essencialmente em relação ao parâmetro de escala c. Foram analisadas diversas trajetórias para diferentes valores do parâmetro de escala. Para isso, com o intuito de comparar a eficiência da varredura do terreno, foi determinado o número de iterações nas quais o vigilante cobriria o terreno satisfatoriamente. $\mathrm{O}$ cobrimento satisfatório do terreno foi considerado como sendo aquele no qual o vigilante realizaria um número mínimo de passagens em cada sub-terreno (duas passagens em cada sub-terreno). As figuras abaixo mostram as trajetórias para alguns valores do parâmetro de escala:

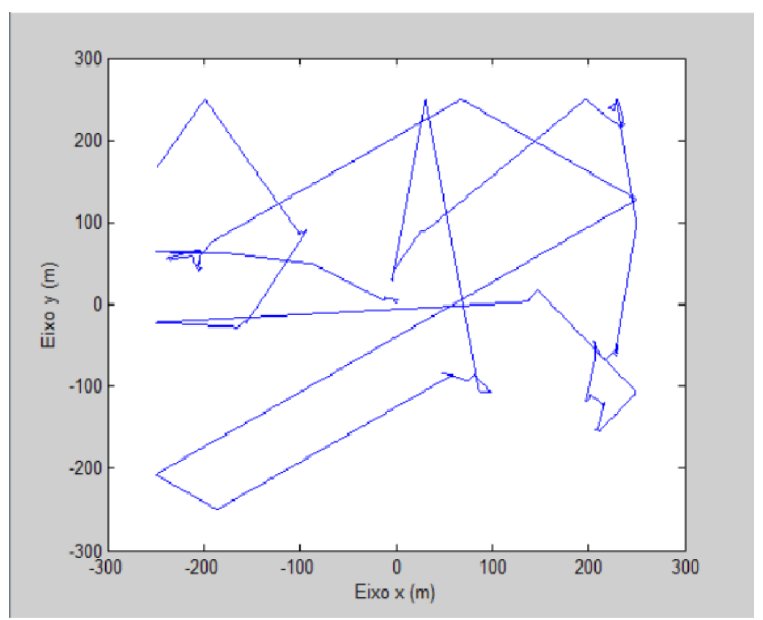

Figura 2: Trajetória do robô pela área delimitada para $\mathrm{c}=1$.

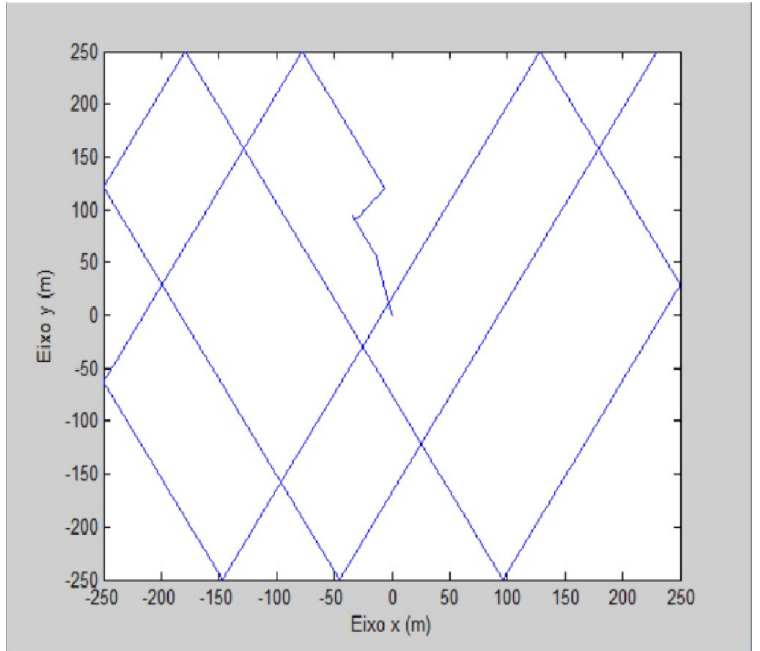

Figura 3: Trajetória do robô pela área delimitada para $\mathrm{c}=7,5$.

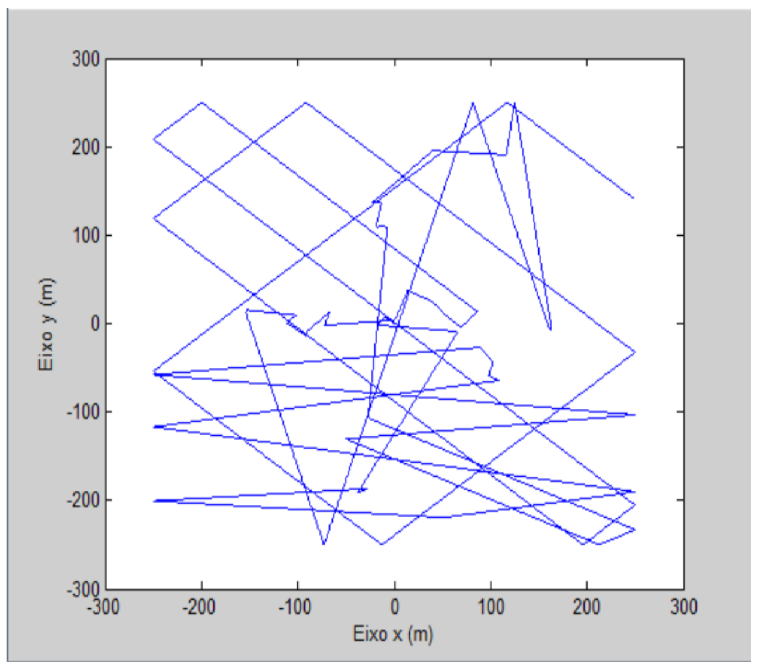

Figura 4: Trajetória do robô pela área delimitada para $\mathrm{c}=15$.

A partir dos resultados mostrados nas figuras 2 , 3 e 4, observa-se que a cobertura está relacionada à variação do parâmetro de escala. Com o aumento deste, os seguimentos de reta que compõem a trajetória do vigilante em cada caso se tornam maiores. Para um mesmo valor da função de lévy, com valores do parâmetro de escala distintos, o valor da distância de caminhada correspondente é maior para valores maiores do parâmetro de escala c. Dessa forma, a chance do vigilante ter que recuar devido ao limite da área é maior (movimento de "zig-zag", conforme fig. 01.) A partir de algumas amostras de trajetórias para os diferentes valores do parâmetro de escala, pode-se tirar uma média do valor total do número de vezes em que o robô passou em cada sub-terreno para o cobrimento satisfatório do terreno em cada caso: 
Tabela 1. Valores de passo para diferentes parâmetros de escala

\begin{tabular}{|c|c|c|c|}
\hline & $\mathrm{c}=1$ & $\mathrm{c}=7,5$ & $\mathrm{c}=15$ \\
\hline $\mathrm{i}=1$ & 7340 & 7081 & 4119 \\
\hline $\mathrm{i}=2$ & 4363 & 7229 & 18236 \\
\hline $\mathrm{i}=3$ & 6808 & 9770 & 7769 \\
\hline $\mathrm{i}=4$ & 11919 & 12238 & 9928 \\
\hline $\mathrm{i}=5$ & 9280 & 6050 & 6030 \\
\hline $\mathrm{i}=6$ & 10000 & 12332 & 11952 \\
\hline $\mathrm{i}=7$ & 7117 & 7595 & 8547 \\
\hline $\mathrm{i}=8$ & 6653 & 9250 & 15401 \\
\hline $\mathrm{i}=9$ & 10993 & 5812 & 13019 \\
\hline $\mathrm{i}=10$ & 6570 & 9138 & 7549 \\
\hline Média & 6691,5 & 8649,5 & 10255 \\
\hline
\end{tabular}

Mais uma vez, pode ser notado o aumento do número de iterações. Entretanto existe outro fator que deve ser levado em conta na trajetória do vigilante: o ângulo de cada caminhada. Vale ressaltar que o ângulo de uma determinada caminhada do vigilante é definido como o arco-tangente entre a razão das diferenças dos valores das coordenadas $\mathrm{x}$ e y respectivamente em dois instantes consecutivos da seguinte forma:

$$
\theta_{\mathrm{i}}=\operatorname{arctg}\left[\left(\mathrm{Y}_{\mathrm{i}+1}-\mathrm{Y}_{\mathrm{i}}\right) /\left(\mathrm{X}_{\mathrm{i}+1}-\mathrm{X}_{\mathrm{i}}\right)\right]
$$

Sendo esta uma variável independente da variável aleatória (comprimento de caminhada), a intenção aqui foi analisar o quão seria melhor o cobrimento do terreno para trajetórias nas quais o vigilante assumiria um único valor de ângulo, caso o robô viesse a percorrer um comprimento de trajetória demasiado grande, de forma a percorrê-lo em movimentos de "zig-zag" devido aos limites da área. Assim sendo, definiu-se aqui novamente um número satisfatório de passagens que o mesmo deveria realizar em cada subárea do terreno, com o intuito de verificar o valor do comprimento de trajetória para cada ângulo. Uma vez que o robô descreve neste caso um movimento de "zig-zag" devido à condição limitante, o ponto de partida para determinar o comprimento é irrelevante. Assim sendo, partindo de um dos vértices do terreno, foi realiza uma simulação que correlacionou o valor do ângulo com o comprimento de trajetória para um número de duas passagens em cada subárea do terreno. A figura seguinte mostra tal relação:

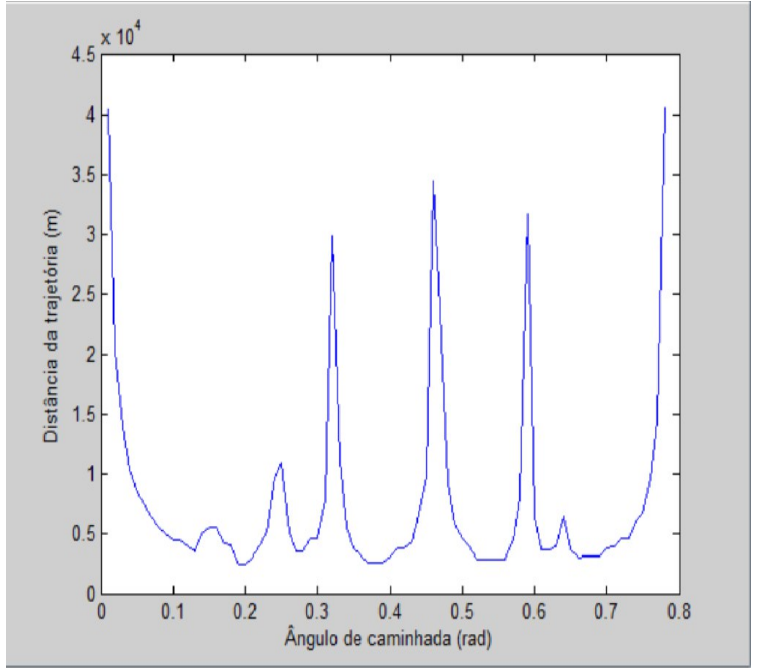

Figura 5: Comprimento de trajetória do robô para valores de ângulo em cobrimento do terreno.

O que pode ser visto aqui é que para três valores de ângulo de trajetória o comprimento da trajetória de cobrimento do terreno possuirá valores de pico. Os ângulos que correspondem a estes valores são de $0,32,0,46$ e 0,60 radianos. Outro fato que pode ser notado, é que para os valores de ângulo de 0 e $\pi / 4$ o comprimento da trajetória tende ao infinito. Isso se deve ao fato de que nestes valores de ângulo o vigilante percorreria uma trajetória infinita: nos valores de $\theta=0, \pi / 2, \pi$ e $3 \pi / 2$ o vigilante iria de um lado para o outro indefinidamente, já que atingindo um dos limites, retornaria com o valor do ângulo complementar, indo e vindo indefinidamente. O mesmo é válido para o valor de $\theta=\pi / 4$ e seus múltiplos: sendo o valor da tangente da diagonal do quadrilátero igual a $\pi / 4$, sucessivas caminhadas no terreno com este valor de ângulo resultariam sempre no mesmo ponto, até que uma nova escolha fosse feita. Dessa forma, este fato evidência uma desvantagem de valores maiores para o parâmetro de escala c: o risco do robô percorrer caminhos em sucessivos movimentos, quando o valor de comprimento for relativamente grande, tornando assim o vigilante previsível e perdendo o efeito desejável da imprevisibilidade da trajetória por um determinado período de tempo. Valores menores do parâmetro de escala proporcionam um melhor cobrimento do terreno (mais rápido) e tornam a preocupação com o ângulo de caminhada menor.

\section{Contribuição deste artigo}

O estudo e a implementação do movimento caótico em um vigilante/buscador mostram-se vantajosos devido a não necessidade do mesmo possuir um mapa de bordo, e, no caso do vigilante, o mesmo não possuir um movimento definido, dificultando a ação de um invasor. 


\section{Conclusão}

A partir dos resultados obtidos pode-se relacionar à variável do parâmetro de escala da função densidade de probabilidade do vôo de Lévy com o cobrimento da área limitada, mostrando um cobrimento de área mais eficiente para valores do parâmetro de escala c maiores. Entretanto, também foi possível notar que com o aumento do mesmo, o movimento pode prover ao vigilante um comportamento previsível por um período de tempo, uma vez que a escolha de comprimentos maiores de cada caminhada faz com que o vigilante percorra caminhos em movimento de "zigzag". Dessa forma, o valor do ângulo numa certa caminhada se torna mais relevante, já que para certos valores do mesmo a probabilidade do vigilante realizar um movimento de "zig-zag" aumento com o aumento do parâmetro de escala.

\section{Agradecimentos}

Os autores agradecem o suporte financeiro do Conselho Nacional de Desenvolvimento Científico e Tecnológico CNPq, da Fundação de Amparo à Pesquisa do Estado de São Paulo - FAPESP, da Coordenação de Aperfeiçoamento de Pessoal de Nível Superior - CAPES, e da Universidade Federal do ABC UFABC.

\section{Referências Bibliográficas}

Edwards, A.; Phillips, R.; Watkins, N.; Freeman, M.; Murphy, E.; et al., "Revisiting Levy flight search patterns of wandering albatrosses, bumblebees and deer", Nature 449, pp. 1044-1048, 2007. DOI: $10.1038 /$ nature06199

Martins-Filho, L.S.; Machado, R.F.; Rocha, R.; Vale, V.S.; "Commanding mobile robots with chaos," in ABCM Symposium Series in Mechatronics, vol. 1, pp. 40-46, ABCM, Rio de Janeiro, Brazil, 2004.

Martins-Filho, L.S.; Macau, E.E.N.; "Patrol Mobile Robots and Chaotic Trajectories", Mathematical Problems in Engineering, Vol. 2007, Article ID 61543, pp. 1-13, 2007.

Nakamura, Y.; Sekiguchi, A.; "The chaotic mobile robot," IEEE Transaction on Robotics andAutomation, vol. 17, no. 6, pp. 898-904, 2001.

Nurzaman, S.G.; Matsumoto, Y.; Nakamura, Y.; Koizumi, S.; Ishiguro, H., "Yuragi-Based Adaptive Searching Behavior in Mobile Robot: From Bacterial Chemotaxis to Levy Walk", Proceedings of the 2008 IEEE International Conference on Robotics and Biomimetics Bangkok, Thailand, February 21 - 26, 2009.
Siegwart, R.; Nourbakhsh, I.R.; "Introduction to Autonomous Mobile Robots", MIT Press, Cambridge, Mass, USA, 2004.

Viswanathan, G.M. et al., "Levy flights and superdiffusion in the context of biological encounters and random searches", Physics and Life Reviews, March 2008. DOI: 10.1016/j.plrev.2008.03.002 